\title{
Basal physiological parameters in domesticated tree shrews (Tupaia belangeri chinensis)
}

\author{
Jing WANG ${ }^{1,3}$, Xin-Li XU ${ }^{1,3}$, Ze-Yang $\mathrm{DING}^{1,4}$, Rong-Rong MAO ${ }^{1}$, Qi-Xin ZHOU ${ }^{1}$, \\ Long-Bao $\mathrm{LV}^{2}$, Li-Ping $\mathrm{WANG}^{1}$, Shuang WANG ${ }^{1}$, Chen $\mathrm{ZHANG}{ }^{1,5}$, Lin $\mathrm{XU}^{1,6}$, Yue-Xiong \\ $\mathrm{YANG}^{1, *}$ \\ 1. Key Laboratory of Animal Models and Human Disease Mechanisms, and Laboratory of Learning and Memory, Kunming Institute of Zoology, \\ Chinese Academy of Sciences, Kunming 650223, China; \\ 2. Kunming Primate Research Center of the Chinese Academy of Sciences, Kunming Institute of Zoology, Chinese Academy of Sciences, Kunming 650223, China; \\ 3. University of Chinese Academy of Sciences, Beijing 100049, China; \\ 4. School of Life Sciences, Anhui University, Hefei 230601, China; \\ 5. Division of Mood Disorders, Shanghai Mental Health Center, Shanghai Jiaotong University School of Medicine, Shanghai 200030, China; \\ 6. Tree Shrew Breeding Center, Kunming Institute of Zoology, the Chinese Academy of Sciences, Kunming 650223, China
}

\begin{abstract}
Establishing non-human primate models of human diseases is an efficient way to narrow the large gap between basic studies and translational medicine. Multifold advantages such as simplicity of breeding, low cost of feeding and facility of operating make the tree shrew an ideal non-human primate model proxy. Additional features like vulnerability to stress and spontaneous diabetic characteristics also indicate that the tree shrew could be a potential new animal model of human diseases. However, basal physiological indexes of tree shrew, especially those related to human disease, have not been systematically reported. Accordingly, we established important basal physiological indexes of domesticated tree shrews including several factors: (1) body weight, (2) core body temperature and rhythm, (3) diet metabolism, (4) locomotor rhythm, (5) electroencephalogram, (6) glycometabolism and (7) serum and urinary hormone level and urinary cortisol rhythm. We compared the physiological parameters of domesticated tree shrew with that of rats and macaques. Results showed that (a) the core body temperature of the tree shrew was $39.59 \pm 0.05^{\circ} \mathrm{C}$, which was higher than that of rats and macaques; (b) Compared with wild tree shrews, with two activity peaks, domesticated tree shrews had only one activity peak from 17:30 to 19:30; (c) Compared with rats, tree shrews had poor carbohydrate metabolism ability; and (d) Urinary cortisol rhythm indicated there were two peaks at 8:00 and 17:00 in domesticated tree shrews, which matched activity peaks in wild tree shrews. These results provided basal physiological indexes for domesticated tree shrews and laid an important foundation for diabetes and stress-related disease models established on tree shrews.
\end{abstract}

Keywords: Domesticated tree shrew; Basal physiological indexes; Rhythm; Glycometabolism; Stress-related hormone

Translational medicine always faces the dilemma of gaps between experimental rodents and humans. Nonhuman primates are considered the best animal models for human diseases. However, macaque models are largely limited by expense, long experimental cycle, expert shortages, and strict animal rights. Thus, it is necessary to find an economical and easily operated nonhuman primate animal model.

The tree shrew (Tupaia belangeri chinensis) is considered a close primate sister, with high genetic similarity to humans (Elliot O, 1971; Janečka et al, 2007).
With fast proliferation and low cost, it is also a potential non-human primate model proxy. Furthermore, many of

\footnotetext{
Received: 11 December 2012; Accepted: 06 February 2013 Foundation items: This work was supported by grants from the Chinese Academy of Sciences (KSCX2-EW-R-12, KSCX2-EW-J-23), the National Natural Science Foundation of China (81171294) and Shanghai Science \& Technology Development Foundation (12140904200).

"Corresponding author, E-mail: yangyx0553@yahoo.com.cn
}

Volume 34 Issue E2 
its features make the tree shrew a suitable model for human disease, especially in relation to stress-related diseases (Cao et al, 2003; Carter, 2007). For example, chronic social defeated depression is well established for tree shrew due to its vulnerability to stress (Fuchs, 2005; Wang et al, 2011). In addition, the existence of spontaneous diabetes in tree shrews makes it possible to establish diabetic models for this species (Liang \& Wu, 2011).

Compared with well-investigated experimental animals, the physiological parameters of $T$. $b$. chinensis have been rarely studied. Here we tested a set of basal physiological indexes for the tree shrew, including daily food and water intake, systolic and diastolic blood pressure, fasting blood glucose, and basal levels of testosterone, estradiol and cortisol. Understanding these parameters could be useful for a variety of research studies using the tree shrew as the model system.

\section{MATERIALS AND METHODS}

\section{Animals}

We used adult tree shrews $(n=92,130-150 \mathrm{~g})$ obtained from a breeding colony at the Animal House Center of the Kunming Institute of Zoology, and Sprague-Dawley (SD) rats $(n=8,250-300 \mathrm{~g})$ obtained from the Kunming Medical University. Animals were individually housed under a $12 \mathrm{~h} / 12 \mathrm{~h}$ dark/light cycle (light, 8:00-20:00; dark, 20:00-8:00) in thermoregulated rooms (T: $25-27{ }^{\circ} \mathrm{C}, \mathrm{RH}: 55 \%-70 \%$ ) with free access to water and food. Animal care and experimental protocols were approved by the Animal Ethics Committee of the Kunming Institute of Zoology, the Chinese Academy of Sciences.

\section{Body-mass and cardiovascular index \\ Body weight, 24-hour diet, locomotion and temperature rhythm}

Eight tree shrews were weighed in the morning. Their food and water intake were measured in metabolism cages. In consideration of food moisture evaporation factors, we also placed a plate of food outside the cage as a control. After six days of 24-hour diet measurement, the tree shrews were returned to their home cage (size: $100 \mathrm{~cm} \times 68 \mathrm{~cm} \times 86 \mathrm{~cm},[\mathrm{w} \times \mathrm{d} \times \mathrm{h}])$ consisting of a glass side. Subjects were videotaped from 8:00 to 20:00 and their movement distance in the vertical plane was recorded by a Noldus EthoVision XT Version 8.0 video tracking system (Wageningen, the Netherlands). Core body temperature was measured using an anal thermometer probe, inserted $2 \mathrm{~cm}$ from the anal sphincter of the tree shrew subjects.

\section{Telemetry monitoring of blood pressure and heart rate}

Blood pressure and heart rate of tree shrews were tested by telemetry monitoring, as described previously (Coolen et al, 2012). In brief, adult tree shrews ( $n=4,3$ male and 1 female) were anaesthetized with pentobarbital sodium $(100 \mathrm{mg} / \mathrm{kg}$, i.p.). Transmitters with radiotelemetric catheters were implanted in enterocoelia to measure blood pressure and heart rate (TAC50-PXT; Data Sciences International, St Paul, MN, U.S.A.). One radiotelemetric catheter was inserted into the abdominal aorta below the renal arteries and fixed into place with tissue adhesive. The implant was then sutured to the abdominal wall, and the abdominal wall and skin incision were sutured with silk suturing. When the animals were awake, blood pressure and heart rate were detected by a PhysiolTel-Receiver (MOPEL RPC-1; Data Sciences International, St Paul, MN, U.S.A.) installed under each individual sleeping-box. Data signals were analyzed using Dataquest A.R.T. t Gold Acquisition, Version 2.20.

\section{Stress-related hormone and sexual hormone Urine cortisol samples}

Urinary cortisol rhythm was tested in tree shrews $(n=30)$. Urine samples were collected each hour from 08:00 to 20:00 and kept at $-20{ }^{\circ} \mathrm{C}$. Samples were measured with an Iodine $\left[{ }^{125} \mathrm{I}\right]$ cortisol radioimmunoassay kit (Beijing North Institute of Biological Technology, China) and $\gamma$ radio-immunity counter (GC-2010, Zonkia, Anhui, China), as per manufacturer's instructions.

\section{Enzyme-Linked Immuno Sorbent Assay (ELISA)}

Whole blood (about $0.2 \mathrm{~mL}$ ) was obtained from the femoral vein of tree shrews $(n=28)$ using a $2 \mathrm{~mL}$ syringe, and centrifuged at $4 \mathrm{C}$ for $8 \mathrm{~min}$ at $5000 \mathrm{r} / \mathrm{min}$. The supernatant was taken and stored at $-20{ }^{\circ} \mathrm{C}$. Sex hormones such as testosterone (T) and estradiol (E2), and serum level of stress-related hormones such as cortisol, norepinephrine (NE), adrenocorticotrophic hormone $(\mathrm{ACTH})$ and corticotrophin releasing hormone $(\mathrm{CRH})$ were assayed using human ELISA kit (Shanghai Li-Chen, China), as per manufacturer's protocols.

\section{Glycometabolism index}

\section{Blood glucose and oral glucose tolerance (OGTT)}

Blood glucose was tested from tail blood using a ONE TOUCH BASIC meter (Lifescan, Inc 2001 Milpitas, CA 95035, USA). Eight tree shrews and eight $\mathrm{SD}$ rats were fasted for $12 \mathrm{~h}$ prior to the experiment. Thereafter, fasting blood glucose (FBG) and 1-hour postprandial blood glucose (PBG) after feeding were tested. In OGTT, animals were given glucose $(2 \mathrm{~g} / \mathrm{kg}, 4$ $\mathrm{g} / \mathrm{kg})$ or saline $(1 \mathrm{~mL} / \mathrm{kg})$ by intragastric administration (i.g.) after 0 point test. Blood glucose was detected at 30 $\mathrm{min}, 60 \mathrm{~min}$, $90 \mathrm{~min}, 120 \mathrm{~min}$, and $360 \mathrm{~min}$ after intragastric treatment. 


\section{Sucrose consumption}

Tree shrews were individually housed $(n=8)$ and were given two bottles containing $200 \mathrm{~mL}$ of water and $200 \mathrm{~mL}$ of sucrose solution. Each concentration $(0.5 \%$, $1 \%, 2 \%, 5 \%, 10 \%$ and $20 \%$ ) was measured once a day, for 2 days. The volumes of consumed water and sucrose solution were recorded.

\section{Electroencephalograms (EEG)}

Three male and three female tree shrews were anaesthetized with pentobarbital sodium $(100 \mathrm{mg} / \mathrm{kg}$, i.p.), ventilated with $95 \% \mathrm{O}_{2}$ and $5 \% \mathrm{CO}_{2}$ and with their core temperature maintained at $37 \pm 0.5^{\circ} \mathrm{C}$. Animals were stereotaxically (RWD Life Science Co., China) implanted with recording electrodes in the hippocampus (15.6 $\mathrm{mm}$ posterior to bregma, $6 \mathrm{~mm}$ lateral to midline, $9.5 \mathrm{~mm}$ below dura) and cerebral cortex (15.6 $\mathrm{mm}$ posterior to bregma, $6 \mathrm{~mm}$ lateral to midline, $2 \mathrm{~mm}$ below dura) on the right hemisphere of the tree shrew brain to measure EEG under anesthetic. Recording electrodes were made by gluing together a pair of twisted Teflon-coated $90 \%$ platinum and $10 \%$ iridium wires $(50$ $\mu \mathrm{m}$ inner diameter, $75 \mu \mathrm{m}$ outer diameter; World Precision Instruments, Sarasota, FL). The EEG signals were amplified and filtered as follows: high-pass filter at $0.1 \mathrm{~Hz}$ and low-pass filter at $2 \mathrm{kHz}$. Sample frequency was $200 \mathrm{~Hz}$. The EEG power spectra were computed by a Fast Fourier Transform routine for $4-\mathrm{s}$ epochs $(0.25 \mathrm{~Hz}$ resolution).

\section{Data analysis}

Data were statistically analyzed using SPSS 19.0. Blood glucose data were analyzed by a Paired-Sample $t$ test and sugar preference concentration test data were analyzed by One-Way ANOVA test, followed by posthoc comparisons (Fisher's LSD test). Significance level was set at $P<0.05$. Data was expressed as mean $\pm S E M$.

\section{RESULTS}

Basal metabolism indexes

Basal metabolism index measurements, body weight, average food and water intake per day, basal levels of $\mathrm{T}$, E2, and cortisol were tested. We also measured the systolic and diastolic blood pressure, heart rate and core body temperature by telemetry monitoring in freely moving tree shrews (Table 1). Compared with published data of other well established laboratory animals, such as rats and macaques, the physiological parameters in our study showed that tree shrews had the highest heart rate and core body temperature, which was likely related to its high activity level. Systolic pressure of tree shrews was much closer to macaques. Also similar to macaques, the main stress-related hormone of tree shrew was cortisol, compared to corticosterone in rats (Table 2).

Table 1 Basal parameters of domestic tree shrews

\begin{tabular}{|c|c|c|}
\hline & Indexes & Tree shrew \\
\hline \multirow[t]{5}{*}{ Body-mass index } & Body weight (g) & $147.90 \pm 1.23$ \\
\hline & Food intake $(\mathrm{g} / \mathrm{d})$ & $116.40 \pm 8.09$ \\
\hline & Water volume $(\mathrm{mL} / \mathrm{d})$ & $4.33 \pm 1.38$ \\
\hline & Core body temperature $\left({ }^{\circ} \mathrm{C}\right)$ & $39.59 \pm 0.05$ \\
\hline & High activity time & $17: 30-19: 30$ \\
\hline \multirow[t]{3}{*}{ Cardiovascular } & Systolic pressure $(\mathrm{kPa})$ & $20.92 \pm 0.04$ \\
\hline & Diastolic pressure $(\mathrm{kPa})$ & $11.81 \pm 2.88$ \\
\hline & Heart rate (beats/min) & $357.00 \pm 31.00$ \\
\hline \multirow[t]{2}{*}{ Sexual hormone } & Testosterone $(\mathrm{T}, \mathrm{ng} / \mathrm{mL})$ & $0.13 \pm 0.01$ \\
\hline & Estradiol $(\mathrm{E} 2, \mathrm{ng} / \mathrm{mL})$ & $42.29 \pm 3.36$ \\
\hline \multirow[t]{3}{*}{ Glycometabolism } & Fasting blood glucose (FBG, mmol/L) & $6.20 \pm 0.84$ \\
\hline & Postprandial blood glucose (PBG, mmol/L) & $10.46 \pm 1.59$ \\
\hline & Sugar sensitive concentration & $5 \%$ \\
\hline \multirow{5}{*}{$\begin{array}{l}\text { Stress-related } \\
\text { hormone }\end{array}$} & Cortisol (ng/mL) & $200.89 \pm 14.24$ \\
\hline & Norepinephrine (NE, ng/mL) & $1.51 \pm 0.03$ \\
\hline & $\begin{array}{l}\text { Adrenocorticotrophic hormone } \\
\text { (ACTH, pg/mL) }\end{array}$ & $57.35 \pm 3.17$ \\
\hline & $\begin{array}{l}\text { Corticotrophin releasing hormone } \\
(\mathrm{CRH}, \mathrm{pg} / \mathrm{mL})\end{array}$ & $64.55 \pm 2.13$ \\
\hline & Urinary cortisol (ng/mL) & $0.63 \pm 0.06$ \\
\hline
\end{tabular}

Table 2 Basal parameters of tree shrew compared with rats and macaques

\begin{tabular}{|c|c|c|c|c|}
\hline & Indexes & Rat & Tree shrew & Macaque \\
\hline Body-mass index & Core body temperature $\left({ }^{\circ} \mathrm{C}\right)$ & $38.80 \pm 0.21$ & $39.59 \pm 0.05$ & $38.70 \pm 0.26$ \\
\hline \multirow[t]{3}{*}{ Cardiovascular } & Systolic pressure $(\mathrm{kPa})$ & $17.29 \pm 1.06$ & $20.92 \pm 0.04$ & $24.10 \pm 3.67$ \\
\hline & Diastolic pressure $(\mathrm{kPa})$ & $11.70 \pm 2.13$ & $11.81 \pm 2.88$ & $18.79 \pm 3.50$ \\
\hline & Heart rate (beats/min) & $289.86 \pm 18.36$ & $357.00 \pm 31.00$ & $157.92 \pm 12.59$ \\
\hline Glycometabolism & $\mathrm{FBG}(\mathrm{mmol} / \mathrm{L})$ & $7.80 \pm 2.67$ & $6.20 \pm 0.84$ & $4.65 \pm 0.53$ \\
\hline \multirow[t]{3}{*}{ Stress-related hormone } & Cortisol (ng/mL) & - & $200.89 \pm 14.24$ & $107.80 \pm 17.60$ \\
\hline & Corticosterone $(\mathrm{ng} / \mathrm{mL})$ & $171.18 \pm 39.98$ & - & - \\
\hline & $\mathrm{ACTH}(\mathrm{pg} / \mathrm{mL})$ & $22.88 \pm 7.41$ & $57.35 \pm 3.17$ & $77.88 \pm 21.34$ \\
\hline
\end{tabular}

Data on rats were from Fan et al (2012), Li et al (2011), Liu et al (2006), Xu et al (2006) and Yu et al (2006), and data on macaques were from Xu et al (2007) and Zhang et al (1998). 


\section{Electroencephalograms (EEG)}

The EEG power spectra of anaesthetized tree shrews were recorded by an electrophysiological recording system (Figure 1A), which showed there were no gender differences in the EEG of domesticated tree shrews. Delta waves $(\delta, 1-4 \mathrm{~Hz})$ were the major waveform in the hippocampus and cerebral cortex (Figure 1B, C).

\section{Circadian rhythm}

For circadian rhythm measurement, tree shrews were videotaped from 8:00 to 20:00 and their movement distance in the vertical plane was recorded. Results showed that tree shrews adapted their behavior in an uncertain environment, with animals fed in the laboratory showing an activity peak period only from 17:30 to 19:30 (Figure 2A). Urine cortisol rhythm was determined by urine sampling at 1-hour intervals from 08:00 to 20:00. Results showed there were two activity peaks at 8:00 and 17:00, respectively, which matched activity peaks in the wild (Figure 2B). Core body temperature was recorded by anal temperature method similar to that often used in rats. During the daytime, minimum body temperature was about $38.7{ }^{\circ} \mathrm{C}$ and maximum was about $40{ }^{\circ} \mathrm{C}$ (Figure 2C).
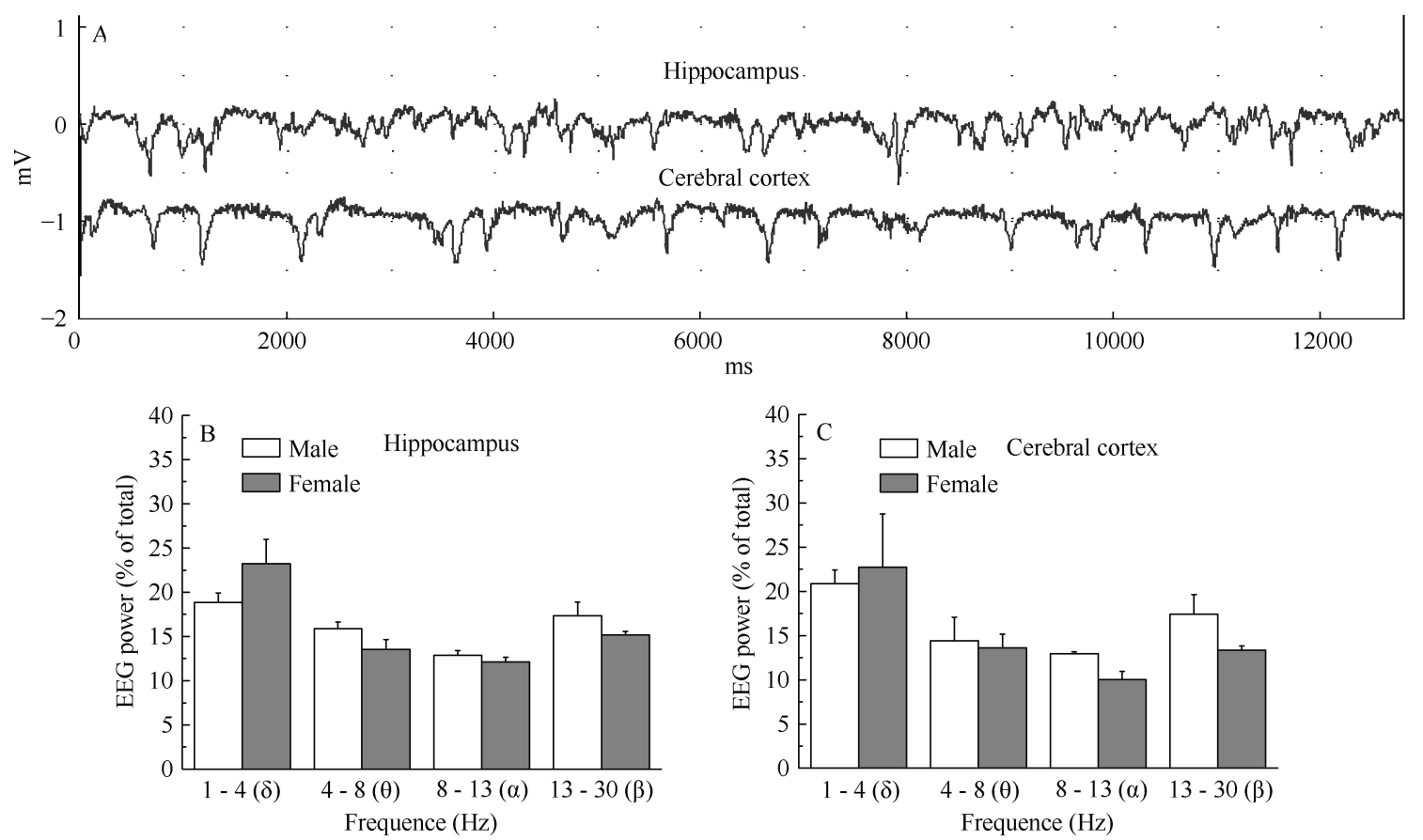

Figure 1 Electroencephalogram of the hippocampus and cerebral cortex in tree shrews

EEG power spectra showed no gender differences in tree shrews. The $\delta$ waves were the major waveform of the hippocampus (B) and cerebral cortex (C) in anaesthetized tree shrews.
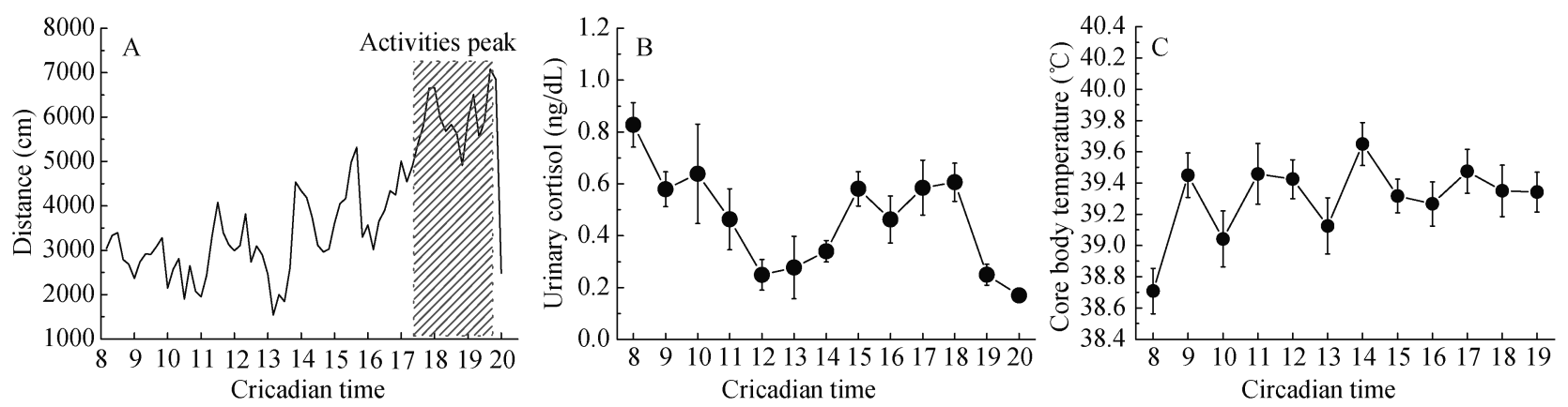

Figure 2 Circadian rhythm of locomotion, urinary cortisol and core body temperature

A: Locomotion activity peak period was from 17:30 to 19:30; B: Urinary cortisol showed two peaks at 8:00 and 17:00 respectively; C: Core body temperature showed a minimum body temperature of $38.7^{\circ} \mathrm{C}$ and maximum of $40^{\circ} \mathrm{C}$.

\section{Glycometabolism}

Blood glucose was tested from tail blood of tree shrews and 1-hour PBG after feeding was double the
FBG (Figure 3A, $t=-3.442, P=0.014$ ). The OGTT results showed that given $2 \mathrm{~g} / \mathrm{kg}$ glucose to fasted animals, the blood glucose of tree shrews increased rapidly for the 

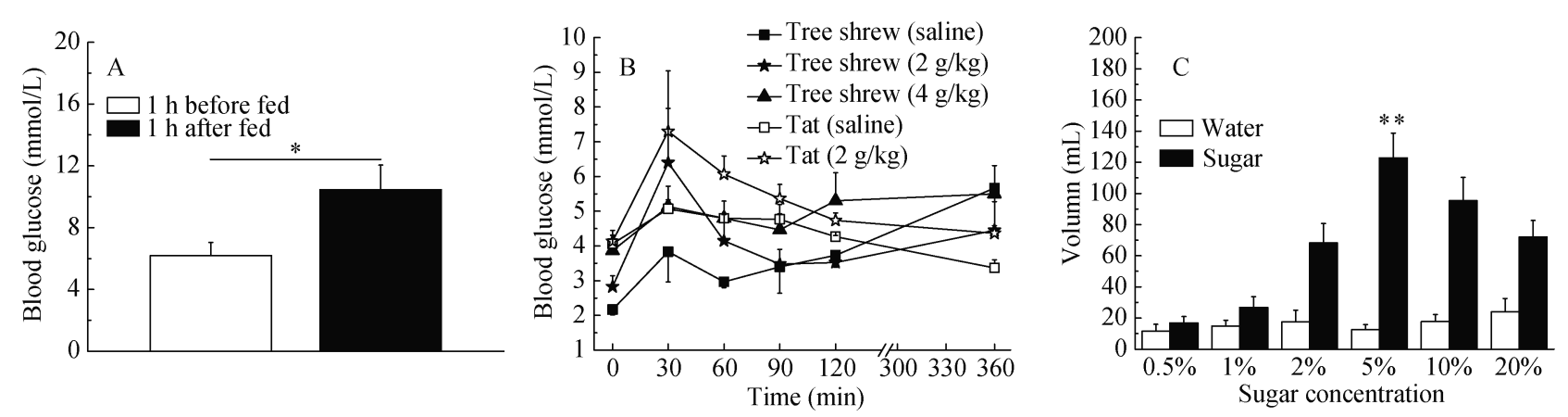

Figure 3 Detection of blood glucose and sugar concentration for tree shrews

A: PBG was significantly higher than FBG; B: Tree shrews and SD rats were given glucose or saline (i.g.) after 0 point test in OGTT. Blood glucose was tested at 30,60, 90, 120 and 360 min after intragastric treatment; C: $5 \%$ was the best sugar concentration among the $0.5,1,2,5,10$ and $20 \% . *: P<0.05, * *: P<0.01$.

first $30 \mathrm{~min}$. After 6 hours metabolizing, the blood glucose level was still high (Figure 3B). The sugar preference concentration test indicated that tree shrews preferred the 5\% sugar concentration (Figure 3C, $\left.F_{[5,88]}=5.964, P=0.000\right)$.

\section{DISCUSSION}

Due to the large differences between rodents and humans, it is extremely difficult to translate basic experimental findings into clinical treatment. Thus, we need new animal models that can reveal mechanisms more closely related to those of human disease. Tree shrews are closely related to primates, and are characterized by fast proliferation, short experiment cycle, and low cost. The brain functions of tree shrews are also very similar to those of humans, and established standardized tree shrew strains could be used to study human disease (Wang et al, 2012). Considering the limited studies previously conducted on $T$. b. chinensis, we determined certain basal physiological indexes for domesticated tree shrews, including body weight, 24hour diet, locomotion and temperature rhythm, systolic and diastolic blood pressure, heart rate, blood glucose, OGTT, sucrose consumption $(0.5 \%, 1 \%, 2 \%, 5 \%, 10 \%$ and $20 \%$ ), basal levels of sex hormones such as testosterone and estradiol and stress-related hormones such as cortisol, norepinephrine, ACTH and CRH. The EEG of the hippocampus and cerebral cortex were also measured.

Compared with rats and macaques, physiological parameters showed that tree shrews had the highest heart rate and core body temperature. Results demonstrated that tree shrews had a high activity level, which makes it an ideal animal model for metabolism studies. Similar to macaques, the main stress-related hormone of tree shrews was cortisol, compared with corticosterone in rats. Circadian rhythm measurement showed that both tree shrews and macaques were diurnal, while the rat was nocturnal. These characteristics suggest that the tree shrew is closely related to non-human primates and is a good animal model for stress-related disease.

For circadian rhythm measurement, domesticated and wild tree shrews had similar cortisol rhythm peaks but different activity peak times. That is, the activity peak period for tree shrews fed in the laboratory showed only one peak from 17:30 to 19:30, but did not show the second peak between 8:00 to 10:00 as seen in wild tree shrews (Fuchs \& Corbach-Söhle, 2010). However, cortisol rhythm in the domestic tree shrew showed two peaks at 8:00 and 17:00, consistent with activity peak times in the wild. The main reason for this difference was because the breeders cleaned the animal house from 8:00 to 10:00, and thus the domesticated tree shrew had adapted to the laboratory environment.

For sucrose consumption, the 5\% concentration was favored by the tree shrews, compared with the $1 \%$ sucrose concentration preferred by rodents (Tye et al, 2013). In the oral glucose tolerance test, tree shrews showed more sensitivity to glucose than rats but demonstrated poor carbohydrate metabolism ability. These might be potential causes for the exhibition of spontaneous diabetes in tree shrews (Liang \& Wu, 2011). Tree shrews would be a good animal model for investigating etiology and pathophysiology of diabetes in future studies.

Our results broaden understanding of the physiological characteristics in tree shrews and also provide detailed reference for future basic scientific research and preclinical study based on tree shrews.

Acknowledgments: We thank the Kunming Institute of Zoology and Gui-Fen XIE for full assistance during the whole experiment. We also thank the Yunnan Planned Parenthood Institute of Science and Technology Biochemical Immune Laboratory for urine sample testing and the Kunming Medical University Hospital for ELISA testing. 


\section{References}

Cao J, Yang EB, Su JJ, Li Y, Chow P. 2003. The tree shrews: adjuncts and alternatives to primates as models for biomedical research. Journal of Medical Primatology, 32(3): 123-130.

Carter AM. 2007. Animal models of human placentation-a review. Placenta, 28: S41-S47.

Coolen A, Hoffmann K, Barf RP, Fuchs E, Meerlo P. 2012. Telemetric study of sleep architecture and sleep homeostasis in the dayactive tree shrew Tupaia belangeri. Sleep, 35(6): 879-888.

Elliot O. 1971. Bibliography of the tree shrews 1780-1969. Primates, 12(3-4): 323-414.

Fan LH, Li D, Liu ML, Fan PH. 2012. Investingation on normal reference range and values of blood physiological and biochemical indexes in clean SD rat. National Medical Frontiers of China, 6(19): 33-34. (in Chinese)

Fuchs E. 2005. Social stress in tree shrews as an animal model of depression: An example of a behavioral model of a CNS disorder. CNS Spectrums, 10(3): 182-190.

Fuchs E, Corbach-Söhle S. 2010. Tree Shrew // Hubrecht R, Kirkwood J. The UFAW Handbook on the Care and Management of Laboratory and Other Research Animals, Eighth Edition. Singapore: Robert Hubrecht and James Kirkwood, 262-275.

Janečka JE, Miller W, Pringle TH, Wiens F, Zitzmann A, Helgen KM, Springer MS, Murphy WJ. 2007. Molecular and genomic data identify the closest living relative of primates. Science, 318(5851): 792-794.

Li HS, Deng X, Luo D, Zhang XF, Li S, Wang Y. 2011. Effect of angiotensin II on heart rate and heart rate variability of rat. Progress of Anatomical Sciences, 17(4): 364-367. (in Chinese)

Liang B, Wu XY. 2011. Progress on nonhuman primate models of diabetes mellitus. Zoological Research, 32(1): 91-96.
Liu W, Qian LJ, Yang ZH, Zhan R, Feng H, Wu L. 2006. The study of neuroendocrine mechanism of depression induced by chronic mild stress. Chinese Journal of Applied Physiology, 22(2): 169-172. (in Chinese)

Tye KM, Mirzabekov JJ, Warden MR, Ferenczi EA, Tsai HC, Finkelstein J, Kim SY, Adhikari A, Thompson KR, Andalman AS, Gunaydin LA, Witten IB, Deisseroth K. 2013. Dopamine neurons modulate neural encoding and expression of depression-related behavior. Nature, 493(7433): 537-541.

Wang J, Zhou QX, Tian M, Yang YX, Xu L. 2011. Tree shrew models: a chronic social defeat model of depression and a one-trial captive conditioning model of learning and memory. Zoological Research, 32(1): $24-30$.

Wang J, Zhou QX, Lv LB, Xu L, Yang YX. 2012. A depression model of social defeat etiology using tree shrews. Zoological Research, 33(1): 92-98. (in Chinese)

Xu LJ, Tang XL, Jiu T, Chen FY. 2007. Biological indicators measurement in laboratory macaques. Laboratory Animal and Comparative Medicine, 27(1): 53-56. (in Chinese)

Xu M, Yu L, Ding YY, Wang YM, Wang SW, Pei JM. 2006. Experimental study on hypotensive effects of cinnamaldehyde in anesthetized rats. Chinese Heart Journal, 18(3): 272-276. (in Chinese)

Yu XK, Shi WZ, Zuo Q, Sun QW, Lu AY. 2006. Effects of exercise on the action scores and plasma adrenocorticotropin and corticosterone in rats under psychological stress. Chinese Journal of Sports Medicine, 25(1): 41-45. (in Chinese)

Zhang LY, Jiang Y, Tian BP, Ma YY, Wang GL. 1998. Study on antipsychological stress of different drugs in macaca mulutta. Journal of Yunnan University (Natural Sciences), 20(3): 197-199. 